\title{
The Study on Performance of Drag Based Water Turbine under Non-immersive Condition
}

\author{
Yiting Pei* and Zekun $\mathrm{Mu}$ \\ 30 School Street, Tilton, NH, US, 03276
}

\begin{abstract}
As electricity plays a paramount role in modern life, the electric power outage is always considered to be a serious problem. For this purpose, our research focuses on improving the efficiency of water turbine. By studying the effects of the number and curvature of turbine blades and stimulating water faucet, the water turbine is expected to effectively boost the rotation under non-immersive condition. The goal of the study is to improve the blade design for higher efficiency of the turbine.

This project presents the research of performance on drag based water turbine. The water turbine, whose whole structure is exposed in the air, in the research is an alternative for conventional immersed water turbine. The size of the water turbine is relatively small compared with industry size. Incoming water can be from any direction. The water turbine displays certain advantageous perspectives. Following the past experiments about this device, a myriad of developments has made over years. The blades of drag based water turbine have been tested with numerous combinations and permutations; thus, the effective choices of blade curvature decided in the research are one-quarter of a circle, one-third of a circle and half a circle, with the arc lengths and thicknesses to be the same. Besides, the number of blades in different experiments are 2, 3, 4 respectively. The incoming flow rate is expected to be constant, even though it fluctuates between 5.45 LPM and 5.95 LPM because of limited experiment equipment. Effects of blade number and curvature are examined, and corresponding conclusions are made.
\end{abstract}

\section{Introduction}

Within recent decades, there is a rise of global concern of the use of non-renewable energy source. Pollution, global warming, and the truth that the non-renewable energy source are running out promote the use of renewable energy to reduce the impact and solve the severe problem. One of the most well-used renewable energy sources is wind/ hydraulic power. Until now, there are two ways of classifying the wind/ water turbine: horizontal or vertical, and lift based and drag based. Recently, a vertical drag based turbine is not commonly seen. In 1929, Savonius patented the Savonius turbine, which is the most commonly used drag based vertical turbine used recently.Although not wildly used, there are many advantages of Savonius turbines. Two of the most significant ones are its simple construction and low cost.

Previously, many study are already made to declare some elements that would influence the efficiency of the Savonius turbine including the effect of end plates, aspect ratio, buckets spacing and overlap, blades numbers and rotor stages, the shapes of the blades, the interference of shaft and other accessories, the influence of Reynold number and turbulence intensity, and the influence of stators when the turbine is completely immersed in the liquid. The end plates, the plainest accessory added on the Savonius rotor, is able to significantly boost the limit of mean power coefficient and maintain the pressure between two sides of one blade at satisfactory level, leading more efficient operation of high tip velocity ratios. $[1,2,3,4]$

According to Vance [1], the aspect ratio of the rotor is proportional relationship between its height and diameter, and 2.0 of the aspect ratio could display splendid performance after the majority of researches.Besides, the effect of large blades spacing would reduce the power of rotor with flow unsatisfactory focus on the concave part of the returning blades; thus, the small buckets spacing is assured for every rotor occurring our experiment. What's more, the influence of the shape and the number of buckets are important parameters in the wind-electric Savonius turbine that have been widely studied. According to Vance[1], Blackwell et al.[5], Saha et al.[2] and Shankar [6] the more buckets could lessen the ranges of values because the extraction of momentum from flow develops along angular positions. Similarly, a blade deflects the flow that could focus on the next blade resulting effect of the performance on following blades, which leads less amount of energy from moving flow converted to mechanical energy through the turbine. [ 2, 5, 6]Therefore, the two-buckets Savonius turbine has the highest maximum mean power coefficient.

Three kinds of shapes of the blade are wildly studied previously -- the "hook" and the twisted bucket. Due to the study of Kamoji et al, rotor with blade shape like hook creates slightly higher moments because the airflow is

* Corresponding author: yiting.pei@tiltonschool.org 
more directed to the tip of the blades with the power coefficient obtained a value of 0.21. [7] Compare to other shapes of blades, the twisted blades seem to have a higher value for average power coefficient. In the study of Saha et al. [2], the value of 0.31 is obtained for the twisted blades, while 0.29 for semicircular profile blades. The helical Savonius rotor is also widely studied. It can be seen the same as having infinite stages for a turbine. However, according to Kamoji et al, it does not create a significant difference from semicircular blades.

According to several previous studies, the interference of shaft is also a significant influence on the turbine. Although it can be used in wind turbines, it causes an efficiency reduction in Savonius turbine. [8] Some other accessories are also used to increase the efficiency of the turbine. Valves that only allow fluid to pass from the concave side of a bucket, for instant, are held to increase on power coefficient for a two-stage turbine with three semicircular profile blades from 0.26 to 0.31 , tested by Saha et al.[2]
The fluid itself, however, was also studied on the effect of turbine efficiency. According to Blackwell et al. [5], the increase of Reynolds number decreases the efficiency of the turbine because of the delayed separation of the boundary layer and therefore reduce the pressure drag on the returning blades. In the study of Akwa [9], it presents that the turbulence intensity also affects the efficiency which the maximum power coefficient decreases as the turbulence coefficient increase.

Another way that is used to provide performance gain is by using stators. Due to the simulation from Mohamed et al.[10], the stator improves the performance gain to at least $30 \%$.

However, there is not much research on the factors that affect turbine efficiency when the turbine is not immersed in the fluid. Therefore, our goal is to figure out whether the curvature of a semicircular bucket and the number of blades would have a different effect on turbine efficiency under non- immersive condition.

Table 1. Parameters of all turbines, T1 to T9 means turbine 1 to turbine 9, blade num means number of blades.

\begin{tabular}{|c|c|c|c|c|c|c|c|c|c|}
\hline & T1 & T2 & T3 & T4 & T5 & T6 & T7 & T8 & T9 \\
\hline curvature & 180 & 180 & 180 & 120 & 120 & 120 & 90 & 90 & 90 \\
\hline blade num & 2 & 3 & 4 & 2 & 3 & 4 & 2 & 3 & 4 \\
\hline
\end{tabular}

\section{Experimental setup}

A water tube is directly connected to the water faucet in the laboratory which creates a maximum water speed of 5.69 LPM. A ZJ-LCD-M flowmeter is connected to the end of the water tube to measure the initial water speed. The angle between the water jet and the blade is adjusted by an (model type) anglemeter to 90 degree. The rotational speed of the turbine is measured by a VICTOR 6236P digital tachometer. Shapr: 3D modeling CAD is used to create the model of the turbines. Then the turbines are made with 3D printers. The weight of all the turbines is controlled at $75 \mathrm{~g}$. All turbines are 5.5 inches tall, and the blades have a height of 3.25 inches. The lengths of all blades are the same, which is 4.712 inches. Turbine 1 has two blades with $90^{\circ}$ curvature shown in Fig 1(a). Here, $90^{\circ}$ curvature means one quarter of a circle. Similarly, $120^{\circ}$ curvature means one third of a circle, and $180^{\circ}$ curvature means half a circle. Parameters of other turbines are listed in Table 1 , where T1 T9 mean turbine $1 \sim$ turbine 9, blade num means the number of blades.

As shown in Figure 1(b), we first set up two iron supports to hold the turbine shaft and the end of the water pipe with a diameter of $3 / 4$ inch. The water pipe is $8 \mathrm{~cm}$ above the turbine blades, and the water impinges at the edge of the blade, as shown in Figure 1(d). The other end of the water pipe is connected to a water jet. Both iron supports are placed in a plastic container to collect the water. The label of the tachometer is put on the edge of one of the blades. Then the valve of the water jet is fully turned on. The water flow rate with is measured with the flowmeter, and the water flow rate is recorded after it is steady. The rotating speed of the turbine with the tachometer is then recorded in the Matlab.
For data analysis, we input the data from the experiment and plot the angular velocity of turbines under different blade number and different curvature. These figures will show the strength of influence on power coefficient by changing the number and the curvature of blades. Furthermore, the plots will display how the power coefficient changes at different number of blades and the same curvature; vice versa, how the power coefficient changes at different curvature and the same blade number.

\section{Results}

Figure 2 shows the performance of the turbine with different numbers and curve of blades under the same water flow rate. As shown in the figure, the turbines with both 3 and 4 blades have the best performance with the largest curvature at $120^{\circ}$, having the rotational speed of $539 \mathrm{rpm}$ and $1027.6 \mathrm{rpm}$. The regressional line of the 3blades turbine has a relatively larger amount of increase in rotational speed and then increase in the rate of change of rotational speed between the curvature of $120^{\circ}$ and $180^{\circ}$. For the turbines with four blades, the rotational speed change in a very small amount, and then increased rapidly when it is a curve of $180^{\circ}$. However, the turbines with 2 blades have the best performance at the curvature of $120^{\circ}$, where it has a rotational speed at $434.3 \mathrm{rpm}$. This is because the efficient time that water hit the blades are shorter at the curvature of $180^{\circ}$ of a two-blade turbine. Therefore, the work done by the water on the blades is relatively smaller than on the blades of the curvature of $120^{\circ}$, which decrease the rotational speed of the turbine. For the turbines with the same curvature of blades, it appears that for non-immersive turbines, the more the number of the turbine, the higher the efficiency within the maximum amount of blades is four. For the turbine with 
the blades that have the curvature of $90^{\circ}$, the maximum rotational speed is $782.8 \mathrm{rpm}$; for the turbine with the blades that have the curvature of $120^{\circ}$, the maximum rotational speed is $781.7 \mathrm{rpm}$; and for the turbine with the blades that have the curvature of $180^{\circ}$, the maximum rotational speed of the turbine is 1027.6 . Whereas for the immersive turbines, the highest rotational speed appears at the blade number of two. This difference between the immersive turbine and non-immersive turbine appears because the turbine's performance is influenced by the water flow after the water hit the blades, while the water flow does not have a significant influence on nonimmersive turbines.

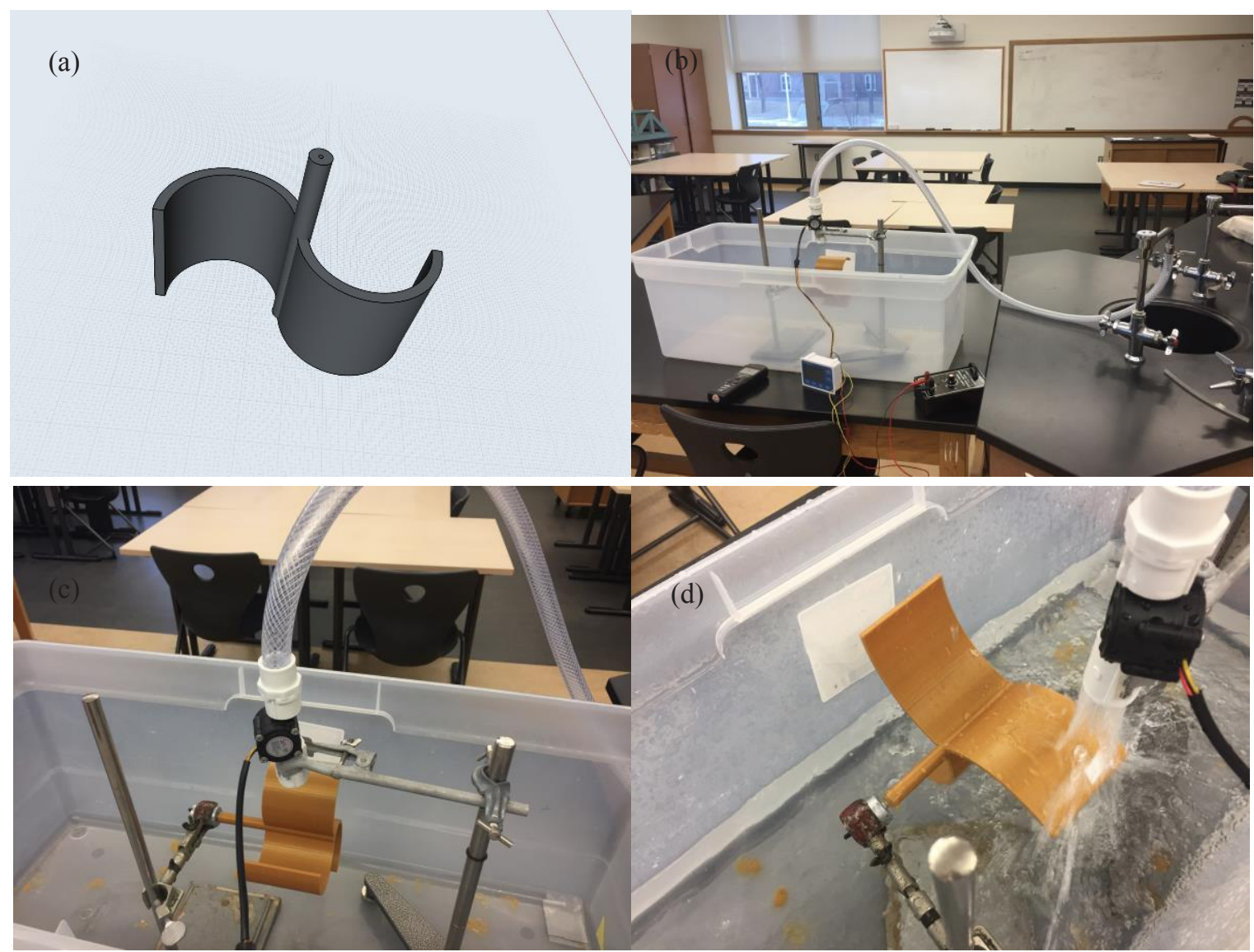

Fig.1. (a) CAD model of turbine 1, (b) experiment setup, (c) a closer view of experiment setup, (d) a snapshot of experiment, which shows the water flow impinges on the outer part of blades.

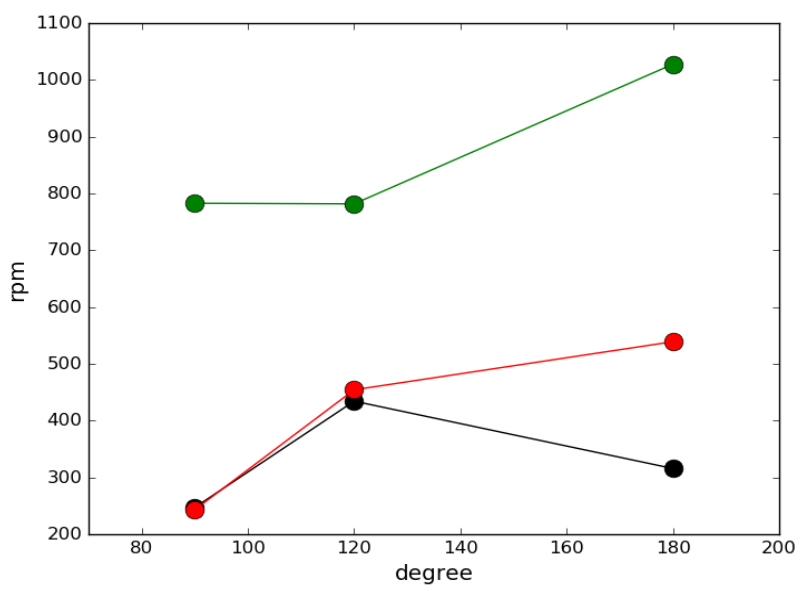

2 blades

3 blades

4 blades

Fig. 2. Performance of the turbine with different numbers and curve of blades. 


\section{Conclusion}

In our study, the performance of turbines with different blades with various numbers and curvature is observed under the same initial water flow rate experimentally, and a graph is created to demonstrate the relationship between them and the power coefficient by measuring the rotational speed of the blades. Due to the observation, we conclude that with the same amount of blades for each turbine, the larger the curvature of the blades, the higher the power coefficient they have except for the two-blade turbines. For two-blade turbines, the highest power coefficient occurs at where the curvature is $120^{\circ}$, which the rotational speed is $434.3 \mathrm{rpm}$. This is because when the curvature is $120^{\circ}$, the effective time of hitting the blades is larger, and therefore the work done on the turbine is larger. For the same curvature, different from immersive turbine where two-blade turbine has the best performance due to the influence of water flow, the larger number of the blades, the higher the rotational speed is. Above all, the best-performed turbine among our experiment sets is the turbine with four blades and $180^{\circ}$ curvature, which have a rotational speed of 1027.6.

\section{References}

1. W. Vance Vert. Axis Wind Roto. - Stat. And Pote. The conference on wind energy conversion systems. 96-102 (1973)
2. UK. Saha, S. Thotla, D. Maity Opti. Desi. Conf. O. Savo. Roto. Thro. Wind Tunn. Expe. J Wind Eng Ind Aerod 96: 1359-75(2008)

3. AJ. Alexander, BP. Holownia Wind Tunn. Test. O. A Savo. Roto. J Ind Aerod 3:343-51(1978)

4. I. Ushiyama, H. Nagai Opti. Desi. Conf And Perf Of Savo. Roto. Wind Eng 12:59-75(1988)

5. BF. Blackwell, RE. Sheldahl, LV. Feltz Wind Tunn. Perf. Data For Two- And Thre.-Buck. Savo. Roto. Sandia Laboratories, USA, Sand 76-0131 under act AT/29-11; 789. (1978)

6. PN. Shankar Deve. Of Vert Axis Wind Turb. Proc Indian Acad Sci.C2(Pt. 1):49-66(1979)

7. MA. Kamoji,SB. Kedare, SV. Prabhu Expe. Inve. On Sing. Stag. Modi. Savo. Roto. Appl Energy 86:106473(2008)

8. MA. Kamoji,SB. Kedare, SV. Prabhu Perf. Test. On Heli. Savo. Roto. Rene. Ener. 34:521-9(2009)

9. JV. Akwa, Savo. Wind Turb. Aero. Anal. Usin. Comp. Flui. Dyna.(2010)

10. MH Mohamed, G Janiga, D. Thévenin, Opti. Blad. Shap. Of A Modi. Savo. Turb. Usin. An Obst. Shie. The Retu. Blad. 52:236-43 (2011) 